\title{
Stochastic Optimization of Braking Energy Storage and Ventilation in a Subway Station
}

\author{
Tristan Rigaut, EFFICACITY, LISIS-COSYS-IFSTTAR, CERMICS-ENPC \\ Pierre Carpentier, UMA-ENSTA \\ Jean Philippe Chancelier, CERMICS-ENPC \\ Michel De Lara, CERMICS-ENPC \\ Julien Waeytens, LISIS-COSYS-IFSTTAR
}

\begin{abstract}
In the Paris subway system, stations represent about one third of the overall energy consumption. Within stations, ventilation is among the top consuming devices; it is operated at maximum airflow all day long, for air quality reasons. In this paper, we present a concept of energy system that displays comparable air quality while consuming much less energy. The system comprises a battery that makes it possible to recover the trains braking energy, arriving under the form of erratic and strong peaks. We propose an energy management system (EMS) that, at short time scale, controls energy flows and ventilation airflow. By using proper optimization algorithms, we manage to match supply with demand, while minimizing energy daily costs. For this purpose, we have designed algorithms that take into account the braking variability. They are based on the so-called Stochastic Dynamic Programming (SDP) mathematical framework. We fairly compare SDP based algorithms with the widespread Model Predictive Control (MPC) ones. First, both SDP and MPC yield energy/money operating savings of the order of one third, compared to the current management without battery (our figure does not include the cost of the battery). Second, depending on the specific design, we observe that SDP outperforms MPC by a few percent, with an easier online numerical implementation.
\end{abstract}

\section{INTRODUCTION}

\section{A. Context}

Apart from train traction, subway stations themselves represent a significant part (one third) of the energy consumption of a subway system in cities like Paris. Fortunately, there is room to reduce their consumption by harvesting some of their unexploited energy potential. We study here the potential energy recovery of a subway station equipped with a battery to recover regenerative braking energy of subways.

Ventilations are among the most significant energy consuming devices in subway stations. One of the reason is because train braking produces a lot of particles that need to be removed by ventilation. By producing regenerative braking energy, trains can dissipate their kinetic energy with a lower bake pads wear. Hence recovering braking energy improves air quality in stations. It might then be useful to control simultaneously a ventilation and a battery to maximize the benefits provided by this interaction.

We present and compare hereby two classes of methods to solve optimal control problems in the presence of uncertainty. The first one is Model Predictive Control which requires only deterministic optimization tools. The second one is Stochastic Dynamic Programming based on Bellman equation. We apply two different flavours of SDP, a state augmentation version to obtain the best possible performance we can achieve with SDP and a more classic one. We make a fair comparison of these methods by Monte Carlo simulation and present the results.

\section{B. Literature}

We survey literature on regenerative braking energy, air quality modelling and energy storage management.

1) Regenerative braking energy: in most recent subway systems, trains already produce regenerative energy when they brake and transmit it to accelerating trains on the same line. However when there is no accelerating train nearby it is not possible to ensure the electrical supply demand equilibrium and regenerative braking is impossible. A comprehensive study of all possible ways to recover that energy is presented in [1]. The authors of [2] conclude that wayside energy storage is relevant to reduce the energy consumption of subway stations. The Southeastern Pennsylvania Transportation Authority (SEPTA) successfully installed wayside batteries to recover braking energy as reported in [3]. SEPTA is about to generalize the project to multiple stations.

2) Air quality in buildings and subway stations: an ANSES report [4] about air quality of underground subway stations states that the concentration of particulate matter whose size is inferior to $10 \mu \mathrm{m}$ (PM10) can be unhealthy for the workers and maybe users. This is mainly due to ferrous PM10 that are generated during braking of the trains as stated in [5] and [6]. Subway stations operators in Paris took measures to monitor the concentration of PM10 [6] that are openly available online. Many studies used Computational Fluid Dynamics technics to model the dispersion of pollutants in subway stations to produce predictive models as did the authors of [7]. These methods are computationally very expensive and could hardly be integrated in an optimization problem without using reduced basis methods [8] that are challenging to implement in dynamic environments such as subway stations. The methods presented in [5] and [9] use zonal models to compute an estimation of the global indoor air quality. These models are much more computationally efficient but require many approximations. The authors of [10] used MPC to control the energy consumption of ventilations and the related climate in a subway station. They estimate that their strategy could save up to $30 \%$ of energy while maintaining the same comfort levels but don't manage an electrical storage simultaneously. 
3) Energy storage management: most of the litterature apply MPC or Two Stage Stochastic Programming techniques to short term operation optimization of energy storage with uncertain supply as observed in [11]. Authors of [12] and [13] present MPC strategies to manage energy in battery and building climate. In [14], [15] and [16] the authors present SDP strategies to control batteries in microgrids. In [17] SDP is applied to smart home management with electricle vehicle battery management. Few papers [18], [19] seem to compare the performance of different stochastic optimal control strategies.

\section{ENERGY SYSTEM MODEL}

We consider the energy system sketched in Figure 1. We present the equations describing its physical evolution in continuous time (denoted by $t$ ): energy storage, Kirchoff laws and air quality. This energy system model will be the basis to simulate different management strategies corresponding to different EMS.

\section{A. Energy storage model}

We use a classical simple model of the dynamics of the energy storage system, with the following variables:

- $s(t)(\%)$, the state of charge of the battery at time $t$;

- $u^{b}(t)(k W)$, the charge $\left(u^{b}(t) \geq 0\right)$ or discharge $\left(u^{b}(t) \leq\right.$ 0 ) power of the battery at time $t$; indeed, we observe on Figure 1 that the battery can draw power on the noational grid or provide power to the station.

The dynamics of the state of charge is 1 .

$$
\frac{d s}{d t}=\rho_{c}\left(u^{b}(t)\right)^{+}+\frac{1}{\rho_{d}}\left(u^{b}(t)\right)^{-},
$$

with charge/discharge efficiencies $\rho_{c}$ and $\rho_{d}$. This simple linear dynamical model is relevant as long as we can ensure, by proper management, that the state $s(t)$ of charge is kept between proper bounds $\underline{s} \leq s(t) \leq \bar{s}$ (like $30 \%$ and $90 \%$ of the capacity), which also ensures a good ageing of the battery.

\section{B. Kirchoff laws}

On Figure 1, we observe that all flows must be balanced at the central node, by Kirchoff laws. The balance equation writes

$$
d(t)+u^{v}(t)+u^{b}(t)=b(t)+u^{r}(t) .
$$

We comment the different terms:

- the station consumes a purely exogenous power $d(t)$ $(k W)$ on the grid at time $t$;

- the ventilations of the station consume a power $u^{v}(t)$ $(k W)$; this energy is controllable and we assume that it can be switched between two modes corresponding to two distinct airflows;

- the trains produce a recoverable power $b(t)(k W)$ on the line;

- the difference $u^{r}(t)=d(t)+u^{v}(t)+u^{b}(t)-b(t)$ is either the trains braking power in excess $\left(u^{r}(t) \leq 0\right)$

${ }^{1}$ We recall that $(x)^{+}=\max (x, 0)$ and $(x)^{-}=\min (0, x)$.

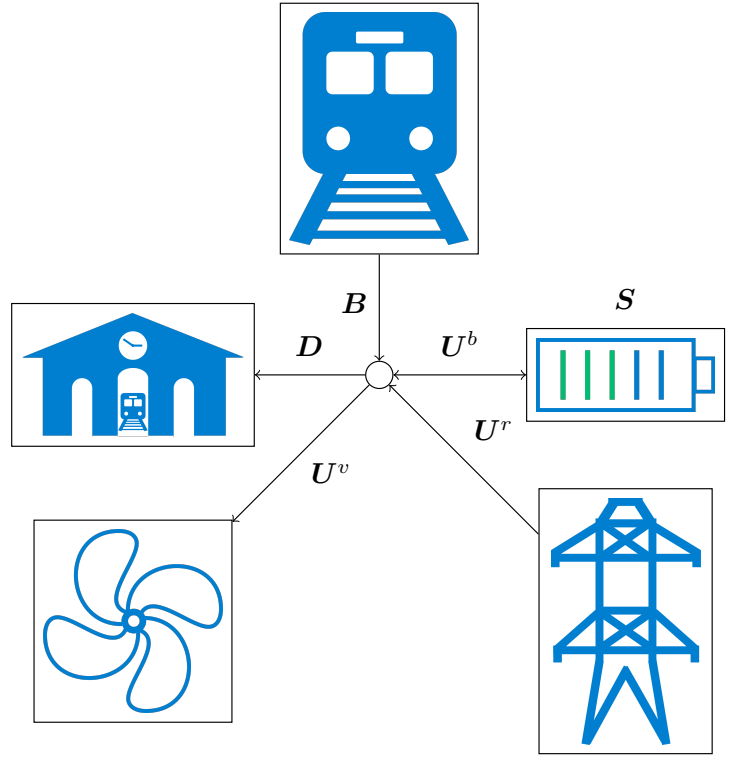

Figure 1. Electrical network representation

that will be wasted (if there is not enough demand, trains brake mechanically instead of electrically), or the power in shortage $\left(u^{r}(t) \geq 0\right)$ that will be drawn on the national grid to satisfy the demand of the station and, possibly, to charge the battery.

\section{Air quality model}

In [9], the authors use a bi-compartiment model in an office building in order to model the deposition/resuspension phenomenon. Due to the lack of data in subway stations to calibrate the surface dynamic model, we consider a simple air mass conservation model, as presented in [5], to model the dynamics of the particulate matters concentration in the subway station air. As in [5], we assume that the floor is always saturated in dust particles so as to ignore the particles surface dynamics. Moreover, we assume that trains arriving in station produce particles by wearing brake pads and wheels, but also by resuspending particles from the floor. We use the model presented in [5] to model the relation between trains arrivals and particles generation in the air.

The dynamical equation for the PM10 concentration in the station is

$$
\begin{aligned}
\frac{d c}{d t} & =\alpha n(t)^{2} \\
& +\left(\frac{\rho_{v}}{v} u^{v}(t)+\beta n(t)\right)\left(c^{o}(t)-c(t)\right)-\delta c(t),
\end{aligned}
$$

with the following notations:

- $c(t)\left(\mu \mathrm{g} / \mathrm{m}^{3}\right)$, PM10 concentration in the station air;

- $c^{o}(t)\left(\mu \mathrm{g} / \mathrm{m}^{3}\right)$, exogenous PM10 concentration outside the station;

- $v\left(m^{3}\right)$, volume of the station assimilated as a single zone;

- $n(t)\left(h^{-1}\right)$, number of arriving trains per hour;

- $\alpha\left(\mu g h / m^{3}\right)$, apparent generation rate of particles by braking trains; 
- $\delta\left(h^{-1}\right)$, apparent deposition rate of particules;

- $\beta$, apparent train contribution rate to natural ventilation;

- $\rho_{v}\left(m^{3} / k w h\right)$, global energy efficiency of the ventilations.

\section{Considerations on numerical simulations}

The equations (1) and (3) form a system of ordinary differential equations. We tested ${ }^{2}$ that a forward Euler resolution with $T_{0}=24 h$ and $\Delta=2$ min coincides with a 5 th order Tsitouras method using adaptative timestepping with a mean error of $0.06 \pm 0.09 \%$. This makes it possible to simulate the energy system, driven by given ventilation and battery control strategies, using a simple discrete time dynamical model.

\section{OPTIMIZATION PROBLEM STATEMENT}

Once we dispose of the energy system dynamical model, we can envisage to simulate different management strategies and to compare them. They are compared with respect to the daily costs that they induce, while respecting constraints. To make this statement more formal and precise, we now formulate a mathematical optimization problem, under the form of a stochastic optimal control problem.

\section{A. Decisions are taken at discrete times}

By contrast with the energy system model developed in Sect. II. where time is continuous, we adopt a discrete time frame because decisions are made at discrete steps. Indeed, we consider a subway station grid equipped with a hierarchical control architecture, as in most microgrids [22], that needs time to compute and implement a decision. Decisions are produced every $\Delta=2$ minutes, over an horizon $T_{0}=24 h$; then, they are sent to local controllers that make decisions at a faster pace.

To make the connection with the variable indexed by continuous time in Sect. II we adopt the following convention: for any variable $x$, we put $x_{t}=x(t \Delta)$ for $t=0, \ldots, T=\frac{T_{0}}{\Delta}$. In other words, $x_{t}$ denotes the value of the variable $x$ at the beginning of the time interval $[t, t+\Delta[$. This dicretization is compatible with the one discussed in II-D

\section{B. Uncertainties are modelled as random variables}

We write random variables in capital bold letters, like $\boldsymbol{Z}$, to distinguish them from deterministic variables $z$.

We model energy demand $D_{t}$ and trains braking energy production $\boldsymbol{B}_{t}$, defined when stating the balance equation (2), as random variables. We do the same for the number $N_{t}$ of trains arrivals per hour and for the outside air quality $\boldsymbol{C}_{t}^{o}$, both defined when stating the dynamical equation (3) for the PM10 concentration in the station.

In the end, we define, for $t=0, \ldots, T$, the vector of uncertainties at time step $t$

$$
\boldsymbol{W}_{t}=\left(\boldsymbol{D}_{t}, \boldsymbol{B}_{t}, \boldsymbol{N}_{t}, \boldsymbol{C}_{t}^{o}\right)^{\top} .
$$

We call $\boldsymbol{W}_{t}$ the noise at time $t$, that is, the uncertainties materialized at the end of the time interval $[t-\Delta, t)$. The noise $\boldsymbol{W}_{t}$ takes value in the set $\mathbb{W}_{t}=\mathbb{R}^{4}$.

\footnotetext{
${ }^{2}$ We used the Julia [20] package DifferentialEquations.j1 [21].
}

\section{Control variables are modelled as random variables}

As time goes on, the noise variables $\boldsymbol{W}_{t}$ are progressively unfolded and made available to the decision-maker. This is why, as decisions depend on observations in a stochastic optimal control problem, decision variables are random variables: the variables in Sect. $\amalg$ will now become random variables in capital bold letters.

At time step $t$, at the beginning of the time interval $[t, t+\Delta[$, the decision-maker takes two decisions: the battery charge/discharge power $\boldsymbol{U}_{t}^{b}$ and the ventilation power $\boldsymbol{U}_{t}^{v}$. Then, at the end of the time interval $[t, t+\Delta[$ ), the decisionmaker selects the power $\boldsymbol{U}_{t+1}^{r}$, drawn from the national grid, to react to the uncertainties $\boldsymbol{D}_{t+1}$ (demand) and $\boldsymbol{B}_{t+1}$ (braking energy) and to ensure the supply demand balance in the grid. This is made possible by a controlled DC/DC converter and supercapacitors that are not modelled in this problem. From the (balance equation) constraint (2):

$$
\boldsymbol{U}_{t+1}^{r}=\boldsymbol{D}_{t+1}+\boldsymbol{U}_{t}^{v}+\boldsymbol{U}_{t}^{b}-\boldsymbol{B}_{t+1} .
$$

We group the two decision/control variables in a vector:

$$
\boldsymbol{U}_{t}=\left(\boldsymbol{U}_{t}^{b}, \boldsymbol{U}_{t}^{v}\right) .
$$

We call $\mathbb{U}_{t}=\mathbb{R}^{2}$ the set in which the controls take their values.

\section{Non-anticipativity constraints for control variables}

To express the fact that the decision-maker (here the EMS) cannot anticipate on the future realizations of the noise, we introduce $\mathcal{F}_{t}$, the sigma algebra generated by all the past noises up to time $t$ :

$$
\mathcal{F}_{t}=\sigma\left(\boldsymbol{W}_{0}, \ldots, \boldsymbol{W}_{t}\right) \text {. }
$$

The increasing sequence $\left(\mathcal{F}_{0}, \ldots, \mathcal{F}_{T}\right)$ is the natural filtration used to model the information flow of the problem. The algebraic non-anticipativity constraint

$$
\sigma\left(\boldsymbol{U}_{t}\right) \subset \mathcal{F}_{t}
$$

expresses the fact that the decision can only be made knowing no more than the past uncertainties [23, chap. 4].

We say that the controls satisfying (8) are $\mathcal{F}_{t}$-measurable. Throughout the paper, a random variable $Z_{t}$ indexed by $t$ is, by convention, $\mathcal{F}_{t}$-measurable, that is, $\sigma\left(\boldsymbol{Z}_{t}\right) \subset \mathcal{F}_{t}$.

\section{E. State and dynamics}

In the energy system model developed in Sect. III the equations (1) and (3) form a system of ordinary differential equations. This is why we introduce two state variables, the state of charge $s_{t}$ and the PM10 concentration $c_{t}$, making thus a two-dimensional state variable

$$
\boldsymbol{X}_{t}=\left(\boldsymbol{S}_{t}, \boldsymbol{C}_{t}\right)^{\top} \text {. }
$$

We call $\mathbb{X}_{t}=\mathbb{R}^{2}$ the state space where the state takes its values.

By sampling the continuous time differential equations (1) and (3) at discrete time steps, and by considering that the control variables are piecewise constant between two steps, we 
can define a discrete time dynamics $f_{t}: \mathbb{X}_{t} \times \mathbb{U}_{t} \times \mathbb{W}_{t+1} \rightarrow$ $\mathbb{X}_{t+1}$. It is such that

$$
\boldsymbol{X}_{t+1}=f_{t}\left(\boldsymbol{X}_{t}, \boldsymbol{U}_{t}, \boldsymbol{W}_{t+1}\right)=\left(\begin{array}{c}
f_{t}^{s}\left(\boldsymbol{X}_{t}, \boldsymbol{U}_{t}, \boldsymbol{W}_{t+1}\right) \\
f_{t}^{c}\left(\boldsymbol{X}_{t}, \boldsymbol{U}_{t}, \boldsymbol{W}_{t+1}\right)
\end{array}\right)
$$

where

$$
\begin{aligned}
& f_{t}^{s}\left(x_{t}, u_{t}, w_{t+1}\right)=s_{t} \\
& +\Delta\left(\rho_{c}\left(u_{t}^{b}\right)^{+}+\rho_{d}^{-1}\left(u_{t}^{b}\right)^{-}\right) \\
& f_{t}^{c}\left(x_{t}, u_{t}, w_{t+1}\right)=c_{t}-\Delta \delta c_{t}+\Delta \alpha n_{t+1}^{2} \\
& +\Delta\left(\frac{\rho_{v}}{v} u_{t}^{v}+\beta n_{t+1}\right)\left(c_{t+1}^{o}-c_{t}\right) .
\end{aligned}
$$

\section{F. Bound constraints on the state and control variables}

As stated when writing the dynamics of the state of charge in (1), the state of charge has to be kept bounded

$$
\underline{s} \leq \boldsymbol{S}_{t} \leq \bar{s} .
$$

The ventilation airflow can swith between two values, leading to the binary constraint

$$
\boldsymbol{U}_{t}^{v} \in\left\{\underline{u^{v}}, \overline{u^{v}}\right\},
$$

and the charge/discharge power is limited, leading to the box constraint

$$
\underline{u^{b}} \leq \boldsymbol{U}_{t}^{b} \leq \overline{u^{b}} .
$$

The bound constraints (12)-(13)-(14), on the state and control variables, can be summed in the synthetic expression

$$
\left(\boldsymbol{X}_{t}, \boldsymbol{U}_{t}\right) \in B_{t} \subset \mathbb{X}_{t} \times \mathbb{U}_{t}
$$

\section{G. The objective is an expected daily cost}

We consider the following criterion to be minimized:

$$
\mathbb{E}\left[\sum_{t=0}^{T-1} p_{t+1}\left(\boldsymbol{U}_{t+1}^{r}\right)^{+}+\lambda \boldsymbol{C}_{t+1}\right] .
$$

We now comment each term.

The term $\mathbb{E}$ stands for the mathematical expectation. By the law of large numbers, mimimizing the mathematical expectation of costs ensures that the system will perform at its best over many days.

Inside the expectation, the sum over time represents the cumulated costs. Those are a mix of two terms.

First, at every time step $t$, we pay the electricity consumed on the national grid between $t-\Delta$ and $t$. We call $p_{t}(€ / k W)$ the cost of electricity per $k W$ between $t-\Delta$ and $t$, that we assume to be deterministic. Therefore we pay $p_{t} \times\left(\boldsymbol{U}_{t}^{r}\right)^{+}(€)$ at time $t$.

Second, we give a price of discomfort relative to air quality. Ideally, we would like to keep $T^{-1} \mathbb{E}\left(\sum_{t=1}^{T} \boldsymbol{C}_{t}\right)$, the expected mean of particles concentration over a day, bounded. Indeed, this is the indicator used by the World Health Organization for its PM concentration guidelines [24]. To handle this constraint, we fix a marginal price $\left.\lambda\left(€ \mathrm{~m}^{3} / \mu \mathrm{g}\right)\right)$ of discomfort associated with this ideal constraint. We have fixed this parameter by trials and errors, after solving the problem for different values of $\lambda$. The cost of discomfort is then $\lambda \times C_{t}(€)$.

Finally, from (16) and (5), we define the instantaneous cost $L_{t}: \mathbb{X}_{t} \times \mathbb{U}_{t} \times \mathbb{W}_{t+1} \rightarrow \mathbb{R}$ by

$$
L_{t}\left(x_{t}, u_{t}, w_{t+1}\right)=p_{t}\left(d_{t+1}+u_{t}^{v}+u_{t}^{b}-b_{t+1}\right)^{+}+\lambda c_{t+1} .
$$

\section{H. Stochastic optimal control problem formulation}

The EMS problem writes as a general Stochastic Optimal Control (SOC) [23] problem in a risk neutral (expectation) setting

$$
\begin{array}{rl}
\min _{\boldsymbol{X}, \boldsymbol{U}} & \mathbb{E}\left[\sum_{t=0}^{T-1} L_{t}\left(\boldsymbol{X}_{t}, \boldsymbol{U}_{t}, \boldsymbol{W}_{t+1}\right)+K\left(\boldsymbol{X}_{T}\right)\right] \\
\text { s.t } & \boldsymbol{X}_{t+1}=f_{t}\left(\boldsymbol{X}_{t}, \boldsymbol{U}_{t}, \boldsymbol{W}_{t+1}\right) \\
& \left(\boldsymbol{X}_{t}, \boldsymbol{U}_{t}\right) \in B_{t} \\
& \sigma\left(\boldsymbol{U}_{t}\right) \subset \mathcal{F}_{t}
\end{array}
$$

where $K$ is as final cost function - which is 0 in our case, as we are indifferent of the state of charge at the end of the day.

\section{COMPUTATION OF ONLINE CONTROL STRATEGIES}

The non anticipativity constraint (18d) can be equivalently replaced by its functional counterpart [23, chap. 3, p86]:

$$
\exists \pi_{t}: \mathbb{W}_{0} \times \ldots \times \mathbb{W}_{t} \rightarrow \mathbb{U}_{t}, \boldsymbol{U}_{t}=\pi_{t}\left(\boldsymbol{W}_{0}, \ldots, \boldsymbol{W}_{t}\right) .
$$

The mapping $\pi_{t}$ is called a strategy (more precisely a noise dependent strategy).

In this paper, we restrict the search to solutions among the class of (augmented) state strategies of the form

$$
\pi_{t}: \mathbb{X}_{t} \times \mathbb{W}_{t} \rightarrow \mathbb{U}_{t}, \boldsymbol{U}_{t}=\pi_{t}\left(\boldsymbol{X}_{t}, \boldsymbol{W}_{t}\right)
$$

This is indeed a restriction, as the state $\boldsymbol{X}_{t}$ is, by the iterated dynamics (10), a function of $\left(\boldsymbol{W}_{0}, \ldots, \boldsymbol{W}_{t}\right)$.

In practice, we are not interested in knowing $\pi_{t}\left(x_{t}, w_{t}\right)$ for all possible values of $\left(x_{t}, w_{t}\right)$; we just want to be able to compute, on the fly, the value $u_{t}=\pi_{t}\left(x_{t}, w_{t}\right)$ when, at time $t$, the couple $\left(x_{t}, w_{t}\right)$ materializes. This is why, in section IV-A and section IV-B, we present two methods for the online implementation of strategies. Both methods compute $u_{t}=\pi_{t}\left(x_{t}, w_{t}\right)$ by solving, online, a optimization problem.

\section{A. Model Predictive Control (MPC)}

MPC is often casted in the context of deterministic optimization as it requires only to solve deterministic problems. However it can be often used to solve stochastic optimization problems. At time step $t$, the MPC algorithm takes as inputs the state $x$ of the system and all the previous uncertainties realizations $w_{0}, \ldots, w_{t}$. One way or another, it selects a "forecast" $\left(\tilde{w}_{t+1}, \ldots, \tilde{w}_{T}\right)$ and then solves the following deterministic (open loop) optimal control problem:

$$
\begin{aligned}
\min _{\left(u_{t}, \ldots, u_{T-1}\right)} & \sum_{s=t}^{T-1} L_{s}\left(x_{s}, u_{s}, \tilde{w}_{s+1}\right)+K\left(x_{T}\right) \\
\text { s.t } & x_{s+1}=f_{s}\left(x_{s}, u_{s}, \tilde{w}_{s+1}\right) \\
& \left(x_{s}, u_{s}\right) \in B_{s}, x_{t}=x
\end{aligned}
$$


From the optimal controls $\left(u_{t}, \ldots, u_{T-1}\right)$ thus obtained, the MPC algorithm only keeps the first $\left(\tilde{u}_{t}, \ldots, \tilde{u}_{t+N_{m p c}}\right)$ (we call $N_{m p c}$ the reoptimization step of the MPC). Then, at time $t+$ $N_{m p c}$, the MPC algorithm produces new controls by solving problem 21 starting at $t+N_{m p c}$ with an updated forecast.

As it proves delicate to select a decent forecast for all the remaining time horizon (and as a bad forecast can lead to poor decisions), the online problem horizon $T-1$ in 21a is often cut at $t+h_{t}$, with $h_{t} \geq N_{m p c}$. Thus, one obtains problem 21) where the objective 21a is replaced by $\sum_{s=t}^{t+h_{t}} L_{s}\left(x_{s}, u_{s}, \tilde{w}_{s+1}\right)$.

\section{B. Stochastic Dynamic Programming (SDP) based algorithms}

A major difference of MPC with the SDP methods is that there is no offline computation phase.

1) The offline-online SDPO algorithm encompasses two phases: a backward functional recursion performed offline; a forward online optimization by exhaustive search.

Offline, the SDPO algorithm computes a sequence of functions $\tilde{V}_{t}$ by backward induction as follows:

$$
\begin{aligned}
\tilde{V}_{T}(x) & =K(x) \\
\tilde{V}_{t}(x) & =\min _{u \in \mathbb{U}_{t}} \int_{\mathbb{W}_{t+1}}\left[L_{t}\left(x, u, w_{t+1}\right)+\right. \\
& \left.\tilde{V}_{t+1}\left(f_{t}\left(x, u, w_{t+1}\right)\right)\right] \mu_{t+1}^{o f}\left(d w_{t+1}\right) .
\end{aligned}
$$

Here, each $\mu_{t+1}^{o f}$ is an (offline) probability distribution on the set $\mathbb{W}_{t+1}$. The recursion is often performed by exhaustive search in discretized versions of the state and control spaces, hence requiring interpolation of the functions $V_{t}$. Indeed, $x_{t+1}=f_{t}(x, u, w)$ is not guaranteed to fall on a gridpoint of the discretized version of $\mathbb{X}_{t+1}$.

Online, at time $t$, the SDPO algorithm uses the functions $V_{t}$ and solves (with possibly a refined discretization of the control space $\left.\mathbb{U}_{t}\right)$

$$
\begin{aligned}
u_{t} \in \underset{u \in \mathbb{U}_{t}}{\arg \min } \int_{\mathbb{W}_{t+1}}\left[L_{t}\left(x, u, w_{t+1}\right)+\right. \\
\left.\tilde{V}_{t+1}\left(f_{t}\left(x, u, w_{t+1}\right)\right)\right] \mu_{t+1}^{o n}\left(w_{t}, d w_{t+1}\right) .
\end{aligned}
$$

Here, $\mu_{t+1}^{o n}$ is an (online) conditional probability distribution on the set $\mathbb{W}_{t+1}$, knowing the previous uncertainty $w_{t}$. We choose a conditional distribution depending here only on the last uncertainty realization because we use an order 1 autoregressive model in our numerical experiment. As the online conditional probability distribution $\mu_{t+1}^{o n}$ depends on past uncertainties, this method produces state and noise dependent decisions in real time.

It is well known [25] that the above offline-online SDPO algorithm produces an optimal solution of the SOC problem (18) when i) the random variables $\boldsymbol{W}_{0}, \ldots, \boldsymbol{W}_{T}$ are stagewise independent, ii) $\mu_{t}^{o f}$ is the probability distribution of $\boldsymbol{W}_{t}$, iii) $\mu_{t}^{o n}=\mu_{t}^{o f}$ is the (unconditional) probability distribution of $\boldsymbol{W}_{t}$.

As, in our energy system case, the uncertainties are very likely correlated between successive time steps, they cannot be modelled by stagewise independent noises. Consequently, the strategy provided by the offline-online SDPO algorithm is not guaranteed to be optimal.

2) The offline-online SDPA algorithm follows the offlineonline SDPO structure, but with: the state $x$ replaced by the couple $(x, w)$; the uncertainty $w$ replaced by a new uncertainty $z$.

The dynamics $f_{t}(x, u, w)$ is also replaced by a dynamics $f_{t}^{A}((x, w), u, z)$ of the form

$$
\begin{aligned}
& f_{t}^{A}:\left(\mathbb{X}_{t} \times \mathbb{W}_{t}\right) \times \mathbb{U}_{t} \times \mathbb{Z}_{t+1} \rightarrow\left(\mathbb{X}_{t+1} \times \mathbb{W}_{t+1}\right) \text { where } \\
& f_{t}^{A}((x, w), u, z)=\left(f_{t}\left(x, u, f^{w}(w, z)\right), f^{w}(w, z)\right) .
\end{aligned}
$$

It is straightforward that the above offline-online SDPA algorithm produces an optimal solution of the SOC problem (18) when there exists a stochastic process $\boldsymbol{Z}_{0}, \ldots, \boldsymbol{Z}_{T}$ such that i) the random variables $\boldsymbol{Z}_{0}, \ldots, \boldsymbol{Z}_{T}$ are stagewise independent, ii) $\boldsymbol{W}_{t+1}=f^{w}\left(\boldsymbol{W}_{t}, \boldsymbol{Z}_{t+1}\right)$.

The limit of this state augmentation strategy is the well known curse of dimensionality. The complexity of SDP grows exponentially with the number of state variables. Here, we try to handle a memory lag of one time step; but handling dependency between noises over multiple time steps would be out of reach.

\section{NUMERICAL RESULTS, ASSESSMENT AND DISCUSSION}

In section IV we outlined three methods to compute online strategies. Now, we detail how to simulate them on the energy system model developed in section II and how to compare their expected daily costs.

\section{A. Common data feeding the algorithms}

1) Reference case: we consider a subway station i) where the ventilation is operated at constant airflow $60 \mathrm{~m}^{3} / \mathrm{s}$ ii) which is not equipped with a battery iii) which does not recover regenerative braking. With this ventilation strategy, the mean PM10 concentration over a day is $108 \mu \mathrm{g} / \mathrm{m}^{3}$, while the maximum is $182 \mu \mathrm{g} / \mathrm{m}^{3}$. The consumption of the station over a day is $2.160 M W h$ which costs $161 €$.

By choosing this reference case, our aim is to measure the daily savings made possible by investing into a battery and by adopting one of the three strategies outlined in section IV This is a partial analysis, as we do not consider the costs of investment.

2) Braking energy scenarios for algorithms design: as stated in (4), the problem presents four sources of uncertainty. However, we assume that, in (4), the demand $\boldsymbol{D}_{t}$, the number $N_{t}$ of trains per hour, and the outdoor particles concentration $C_{t}^{o}$ are deterministic in our numerical experiment. Indeed, most of the uncertainty comes from the trains energy recovery and, moreover, we can have pretty accurate forecasts for the variables that we assume deterministic.

A scenario is any possible realization of the noise process $\left(\boldsymbol{W}_{0}, \ldots, \boldsymbol{W}_{T}\right)$ written $\left(w_{0}, \ldots, w_{T}\right)$. For the braking energy, we generated 5,000 so-called optimization scenarios by using a rule, provided in the link in appendix $\mathrm{A}$, calibrated on realistic data. These 5,000 optimization scenarios are the common input provided to all the optimization algorithms, so that they can be used to design the features of each algorithm. 


\section{B. Numerical implementation of the MPC algorithm}

1) Forecast: knowing a realization $w_{t}$ of the noise $\boldsymbol{W}_{t}$, we need to compute a forecast $\left(\tilde{w}_{t+1}, \ldots, \tilde{w}_{T}\right)$ of the future uncertainties. The forecast relies upon the following log$\operatorname{AR}(1)$ mode 3

$$
\log \boldsymbol{W}_{t+1}=a \log \boldsymbol{W}_{t}+\boldsymbol{Z}_{t+1}, \quad \forall t=0, \ldots, T,
$$

with independent residual random variables $\left(\boldsymbol{Z}_{t}\right)_{t=1, \ldots, T}$. The coefficient $a$ and the distribution of the residuals are identified using the 5,000 optimization scenarios.

2) Deterministic problem resolution: MPC requires to solve the determistic problem 21. We present the resolution method, based on a MILP formulation presented in Appendix B

\section{Numerical implementation of the SDP algorithms}

1) The offline-online SDPO algorithm: it requires as input the probability distributions $\mu_{t}^{o f}$, used to compute the functions $V_{t}$ offline, and the conditional probability distributions $\mu_{t}^{o n}$, used to compute the controls online.

- $\mu_{t}^{o f}$ : we fit discrete probability distributions at each time step by quantizing, using k-means algorithm, the values taken by the 5,000 optimization scenarios at this very time step $t$.

- $\mu_{t}^{o n}$ : knowing the realization $w_{t-1}$, we obtain the conditional probability distributions $\mu_{t}^{o n}$ by using the formula $w_{t}=w_{t-1}^{a} \exp \left(z_{t}\right)$ (see 25). From the 5,000 optimization scenarios, we obtain 5,000 values of $z_{t}$, hence 5,000 values of $w_{t}$ by $w_{t}=w_{t-1}^{a} \exp \left(z_{t}\right)$.

2) The offline-online SDPA algorithm: in addition to what is needed for the above SDPO algorithm, it requires as input the new dynamics $f^{w}(w, z)$ ) such that $\boldsymbol{W}_{t+1}=$ $f^{w}\left(\boldsymbol{W}_{t}, \boldsymbol{Z}_{t+1}\right)$. This dynamic is deduced from Equation 25.

\section{Out of sample assessment of strategies}

We have generated 10,000 so-called assessment scenarios, to be used only for the assessment phase.

We take good care to distinguish "optimization scenarios" from "assessment scenarios", as displayed in Figure 2. They are sealed. Optimization scenarios were used to construct items entering the design of the MPC and SDP algorithms. Assessment scenarios will be used to compare the strategies produced by these algorithms. This is what we call out of sample assessment. By this sealing, no algorithm can take advantage of the assessment scenarios to be more fitted to the assessment phase.

The result of the assessment of a given strategy/algorithm is an histogram of all the 10,000 costs obtained along the assessment scenarios.

\section{E. Numerical results}

The computer used has Core i7, 2.2Ghz processor and $8 \mathrm{Go}$ ram + 12 Go swap SSD memory.

\footnotetext{
${ }^{3}$ The $\log$ transform ensures that we produce non negative forecasts.
}
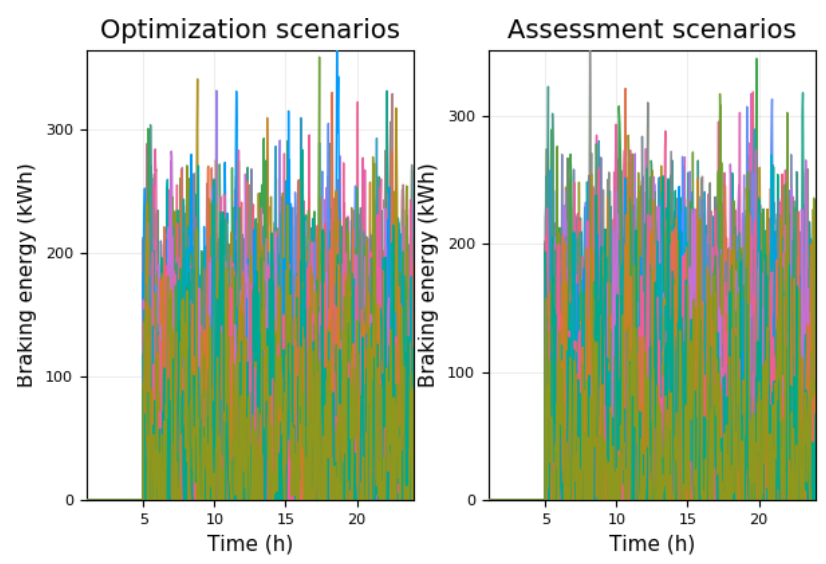

Figure 2. Braking energy scenarios

1) Comparing the algorithms performance: the results 4 are summed up in Table I. We measure the savings with respect to a reference case with no regenerative braking recovered and ventilation at constant maximum speed over the day.

\begin{tabular}{|l|c|c|c|}
\hline Strategy & SDPA & SDPO & MPC \\
\hline Offline time & $3 h 47$ & $0 h 06$ & $0 h 00$ \\
Online time & $4.0 \mathrm{~ms}$ & $0.25 \mathrm{~ms}$ & $8.5 \mathrm{~ms}$ \\
\hline & & & \\
Money savings (€) & $-74.1 \pm 4.87$ & $-73.1 \pm 4.54$ & $-71.1 \pm 4.44$ \\
PM10 $\left(\frac{\mu g}{m^{3}}\right)$ & $106 \pm 0.11$ & $107 \pm 0.11$ & $107 \pm 0.08$ \\
Energy savings $(\mathrm{kWh})$ & $-1050 \pm 69.8$ & $-970 \pm 59.55$ & $-942 \pm 59.2$ \\
\hline
\end{tabular}

Table I

STRATEGIES PERFORMANCES COMPARISON

We observe in Table [I that all algorithms provide close results. As we look in more detail, we see that SDPA outperforms both SDPO and MPC on average for the economic savings, the mean PM10 concentration and the saved energy. However, regarding the economic savings, the differences in mean performance (of order $3 €$ ) are lower than standard deviations (of order $4.5 €$ ), which makes it delicate to conclude. The same analysis goes for the energy savings, although the confidence intervals overlap less.

In fact, the three algorithms can be ranked as follows: SDPA outperforms SDPO that outperforms MPC, for the economic and energy savings (and they are comparable for air quality). To sustain this assertion, one has to look at Figure 3 that represents the distribution of the relative performance gap between MPC and SDPA for the economic savings (a comparable analysis holds for the energy savings).

\footnotetext{
${ }^{4}$ The lower the better, as we minimize costs. Results are \pm the standard deviation.
} 


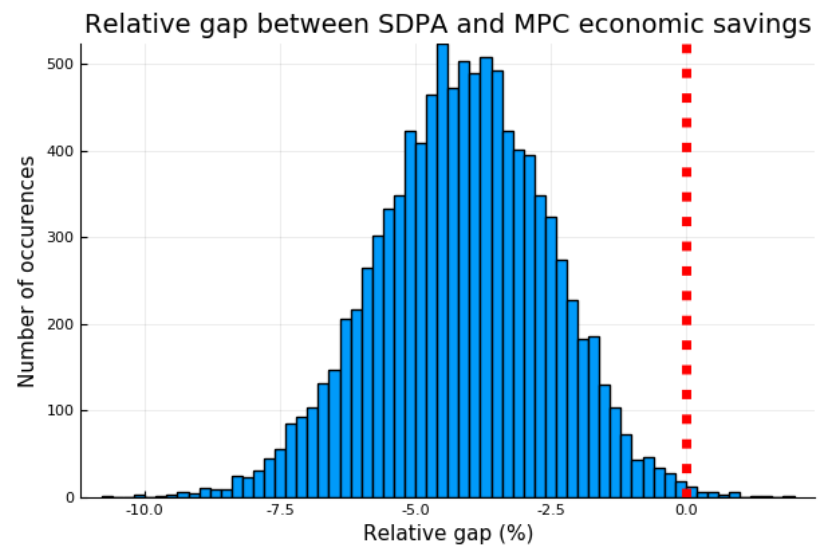

Figure 3. Relative savings gap between SDPA and MPC

On Figure 3, the negative portion of the distribution to the left of the dotted red line is a testimony in favor of SDPA. Our analysis of the assessment scenarios leads to the following observations: i) SDPA outperforms MPC for 9,967 out of the 10,000 scenarios, ii) SDPA outperforms SDPO for 8, 221 out of the 10,000 scenarios, iii) SDPO outperforms MPC for all the scenarios.

Concerning the computation time, Table 1 shows that SDPA requires higher offline computation time than SDPO and MPC. As the online computation time for the three methods is way under 2 minutes, the three methods are implementable in real time (recall that the decision time step is 2 minutes). However, MPC differs from SDP algorithms along the following line: MPC requires to solve a MILP online, so that there is no guarantee to reach the optimum, or a feasible solution, within the prescribed 2 minutes; by contrast, both SDP algorithms only perfom an exhaustive search over all controls in few milliseconds, which we consider safer for critical applications.

2) Energy and air quality results: we display and comment some energy and air quality results based on some of the 10,000 assessment simulations.
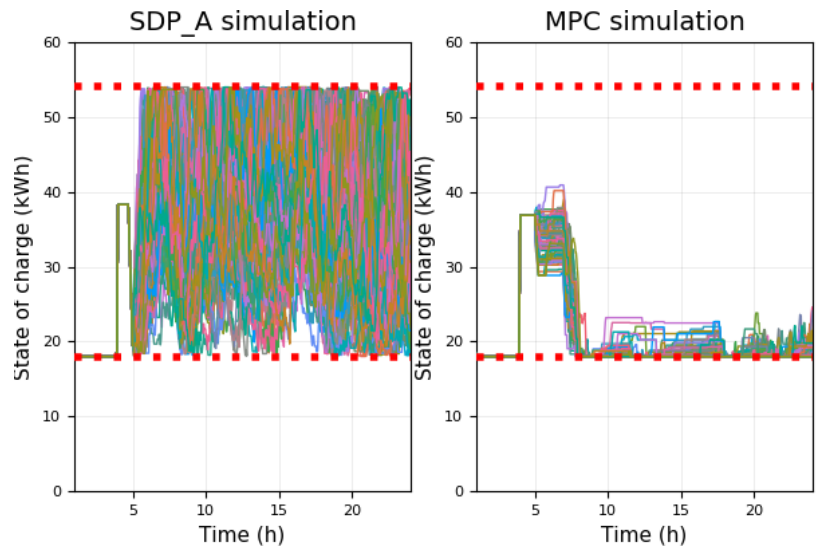

Figure 4. Simulations of the state of charge

Figure 4 displays the state of charge trajectories of the battery on the 10,000 assessment scenarios for SDPA and MPC. We observe that the battery is more intensively operated when using SDPA, illustrating SDPA's ability to recover more energy than MPC.
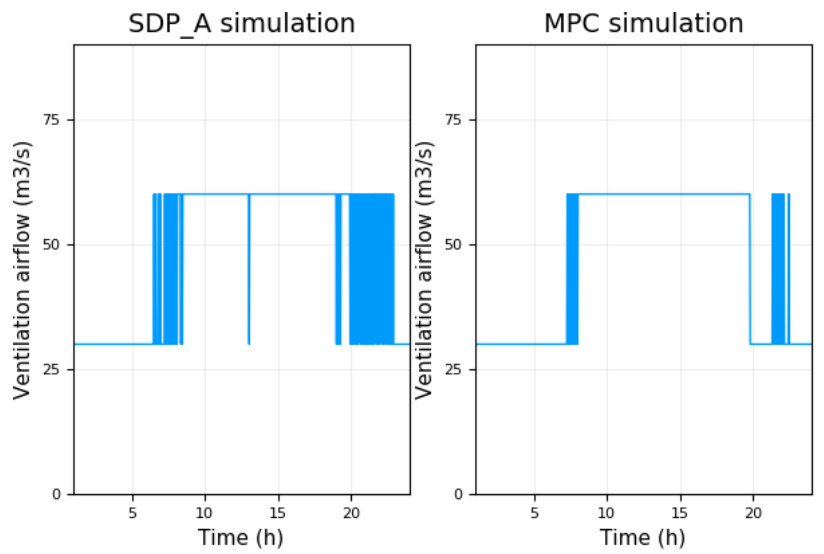

Figure 5. Simulations of the ventilation airflow

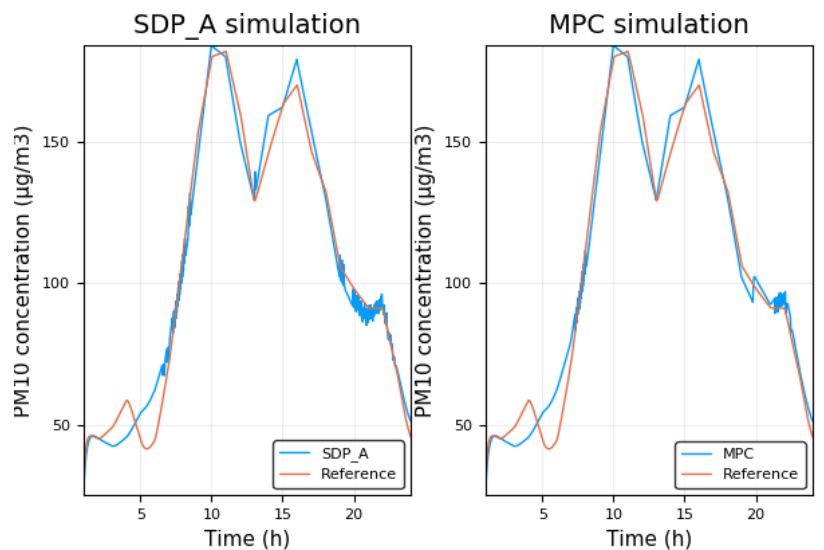

Figure 6. Air quality simulations

Figures 5 and 6 display respectively the controls of the ventilation and the impact on the PM10 concentration over 1 scenario for both SDPA and MPC. We recall that, in the reference case, the ventilation is operated at $60 \mathrm{~m}^{3} / \mathrm{s}$ over the whole day. We observe that both algorithms decrease the consumption of the ventilation while maintaining a similar air quality. 5

\section{CONCLUSIONS AND PERSPECTIVES}

We have presented a subway station energy system, with a battery recovering trains braking and smart control of the ventilations. We have investigated methods to develop and implement an Energy Management System that is able to handle uncertainties related to energy generation. We have discussed the pros and cons of two popular techniques: Stochastic Dynamic Programming (SDP) and Model Predictive Control (MPC). For such a system (with a reasonable number of state variables), we have concluded that SDP is the best choice, even if MPC is a decent alternative. This is not the case in

\footnotetext{
${ }^{5} \mathrm{Had}$ we modeled the particles generation reduction due to braking energy recovery, we would have obtained a sharper decrease in PM10 concentration.
} 
this paper but we recall that MPC could require computationally expensive mathematical programming techniques to solve online deterministic problems.

Our numerical experiments provide encouraging results. It seems that it pays to optimize to improve the energy efficiency and air quality of subway stations. Indeed, as seen on Figures 5 and 6 , the ventilations energy consumption can be decreased without deteriorating the air quality.

Our contribution is a first step towards the analysis of new subway station energy systems. It needs to be completed by an economic analysis that includes the costs of batteries and the practical installation of such systems.

\section{APPENDIX}

\section{A. Data and parameters}

All the data used for the article is available on the following website: https://trigaut.github.io/VentilationArticle.html

\section{B. Deterministic problem resolution}

To solve the MPC deterministic problem, we use mathematical programming techniques by minimizing over states and control variables. The dynamical equations $(11 \mathrm{~b}-(11 \mathrm{a})$ are then simply equality constraints between decision variables. As the ventilation airflow $u_{t}^{v}$ can switch between two modes, one of the decision variables is binary, leading to a Mixed Integer Non Linear Program (MILP). We use two simple tricks to turn 21 into a Mixed Integer Linear Program. The constraint $(11 \mathrm{~b})$ contains the (non linear) product term $u_{t}^{v} \times c_{t}$. To replace this term, we introduce the continuous variables $a_{t}$ and the linear constraints $0 \leq a_{t} \leq \bar{C} \times u_{t}^{v}$ and $c_{t}-\left(1-u_{t}^{v}\right) \bar{C} \leq a_{t} \leq c_{t}$, which ensures that $a_{t}=u_{t}^{v} \times c_{t}$ at optimality.

The constraint 11a contains positive and negative parts of $u_{t}^{b}$, introducing non linearities. To circumvent the problem, we introduce two decision variables, $u_{t}^{b+}$ and $u_{t}^{b-}\left(u_{t}^{b+}=\right.$ $\left(u_{t}^{b}\right)^{+}$and $\left.u_{t}^{b-}=\left(u_{t}^{b}\right)^{-}\right)$, together with the constraint $u_{t}^{b+} \times$ $u_{t}^{b-}=0$. It appears that this latter constraint can be removed as it always satisfied at optimality. Indeed, there is no interest to flow through the battery to reach the demand as the battery efficiency coefficients waste power.

To solve this MILP, we use the Julia package JuMP [26] with the commercial solver Gurobi [27].

\section{REFERENCES}

[1] Arturo González-Gil, Roberto Palacin, and Paul Batty. Sustainable urban rail systems: Strategies and technologies for optimal management of regenerative braking energy. Energy conversion and management, 75:374-388, 2013.

[2] A González-Gil, R Palacin, P Batty, and JP Powell. A systems approach to reduce urban rail energy consumption. Energy Conversion and Management, 80:509-524, 2014.

[3] Andrew J Gillespie, Erik S Johanson, and David T Montvydas. Energy storage in pennsylvania: Septa's novel and innovative integration of emerging smart grid technologies. IEEE Vehicular Technology Magazine, 9(2):76-86, 2014.

[4] Anses. Pollution chimique de l'air des enceintes de transports ferroviaires souterrains et risques sanitaires associés chez les travailleurs. Technical report, Agence nationale de sécurité sanitaire de l'alimentation, de l'environnement et du travail, 2015.
[5] E Walther, M Bogdan, and R Cohen. Modelling of airborne particulate matter concentration in underground stations using a two size-class conservation model. Science of The Total Environment, 607:1313-1319, 2017.

[6] Dorothée Grange and Sabine Host. Pollution de l'air dans les enceintes souterraines de transport ferroviaire et santé. Technical report, Observatoire régional de santé Île-de-France, 2012.

[7] Fernando E. Camelli, Greg Byrne, and Rainald Lhner. Modeling subway air flow using cfd. Tunnelling and Underground Space Technology, 43(Supplement C):20 - 31, 2014.

[8] Christophe Prud'Homme, Dimitrios V. Rovas, Karen Veroy, Luc Machiels, Yvon Maday, Anthony T. Patera, and Gabriel Turinici. Reliable Real-Time Solution of Parametrized Partial Differential Equations: Reduced-Basis Output Bound Methods. Journal of Fluids Engineering, 124(1):70-80, November 2001.

[9] Jing Qian, Andrea R. Ferro, and Kathleen R. Fowler. Estimating the resuspension rate and residence time of indoor particles. Journal of the Air \& Waste Management Association, 58(4):502-516, 2008.

[10] M Vaccarini, Alberto Giretti, LC Tolve, and M Casals. Model predictive energy control of ventilation for underground stations. Energy and buildings, 116:326-340, 2016.

[11] Anthony Papavasiliou, Yuting Mou, Léopold Cambier, and Damien Scieur. Application of stochastic dual dynamic programming to the real-time dispatch of storage under renewable supply uncertainty. IEEE Transactions on Sustainable Energy, 2017.

[12] Alessandra Parisio, Evangelos Rikos, and Luigi Glielmo. A model predictive control approach to microgrid operation optimization. IEEE Transactions on Control Systems Technology, 22(5):1813-1827, 2014.

[13] Peter Pflaum, Mazen Alamir, and Mohamed Yacine Lamoudi. Comparison of a primal and a dual decomposition for distributed MPC in smart districts. In Smart Grid Communications (SmartGridComm), 2014 IEEE International Conference on, pages 55-60. IEEE, 2014.

[14] Benjamin Heymann, J Frédéric Bonnans, Pierre Martinon, Francisco J Silva, Fernando Lanas, and Guillermo Jiménez-Estévez. Continuous optimal control approaches to microgrid energy management. Energy Systems, pages 1-19, 2015.

[15] Benjamin Heymann, J Frédéric Bonnans, Francisco Silva, and Guillermo Jimenez. A stochastic continuous time model for microgrid energy management. In Control Conference (ECC), 2016 European, pages 2084-2089. IEEE, 2016.

[16] Pierre Haessig, Thibaut Kovaltchouk, Bernard Multon, Hamid Ben Ahmed, and Stéphane Lascaud. Computing an optimal control policy for an energy storage. In EuroSciPy 2013, Bruxelles, 2013.

[17] Xiaohua Wu, Xiaosong Hu, Scott Moura, Xiaofeng Yin, and Volker Pickert. Stochastic control of smart home energy management with plug-in electric vehicle battery energy storage and photovoltaic array. Journal of Power Sources, 333:203-212, 2016.

[18] Asbjørn N Riseth, Jeff N Dewynne, and Chris L Farmer. A comparison of control strategies applied to a pricing problem in retail. arXiv preprint arXiv:1710.02044, 2017.

[19] W. B. Powell and S. Meisel. Tutorial on stochastic optimization in energy part ii: An energy storage illustration. IEEE Transactions on Power Systems, 31(2):1468-1475, March 2016.

[20] Jeff Bezanson, Stefan Karpinski, Viral B Shah, and Alan Edelman. Julia: A fast dynamic language for technical computing. arXiv preprint arXiv:1209.5145, 2012.

[21] Christopher Rackauckas and Qing Nie. Differentialequations.j1 - a performant and feature-rich ecosystem for solving differential equations in julia. Journal of Open Research Software, 5(1), 2017.

[22] Daniel E Olivares, Ali Mehrizi-Sani, Amir H Etemadi, Claudio A Cañizares, Reza Iravani, Mehrdad Kazerani, Amir H Hajimiragha, Oriol Gomis-Bellmunt, Maryam Saeedifard, Rodrigo Palma-Behnke, et al. Trends in microgrid control. IEEE Transactions on smart grid, 5(4):1905-1919, 2014.

[23] Pierre Carpentier, Guy Cohen, Jean-Philippe Chancelier, and Michel De Lara. Stochastic Multi-Stage Optimization, volume 75 of Probability Theory and Stochastic Modelling. Springer, 2015.

[24] WHO. Air Quality Guidelines: Global Update 2005. Particulate Matter, Ozone, Nitrogen Dioxide and Sulfur Dioxide. World Health Organization, 2006.

[25] Dimitri P Bertsekas. Dynamic programming and optimal control. Athena Scientific, 1995.

[26] Iain Dunning, Joey Huchette, and Miles Lubin. JuMP: A modeling language for mathematical optimization. SIAM Review, 59(2):295-320, 2017.

[27] Gurobi Optimization Inc. Gurobi Optimizer Reference Manual, 2014. http://www.gurobi.com. 